\title{
Tuning Cellular Biological Functions Through the Controlled Release of NO from a Porous Ti-MOF
}

\author{
Rosana V. Pinto + Sujing Wang ${ }^{+}$, Sergio R. Tavares, João Pires, Fernando Antunes, \\ Alexandre Vimont, Guillaume Clet, Marco Daturi, Guillaume Maurin, Christian Serre,* and
}

Moisés L. Pinto*

\begin{abstract}
Materials for the controlled release of nitric oxide (NO) are of interest for therapeutic applications. However, to date, many suffer from toxicity and stability issues, as well as poor performance. Herein, we propose a new NO adsorption/ release mechanism through the formation of nitrites on the skeleton of a titanium-based metal-organic framework (MOF) that we named MIP-177, featuring a suitable set of properties for such an application: (i) high NO storage capacity $\left(3 \mathrm{umolmg}^{-1}{ }_{\text {solid }}\right)$, (ii) excellent biocompatibility at therapeutic relevant concentrations (no cytotoxicity at $90 \mu \mathrm{g} \mathrm{mL}^{-1}$ for wound healing) due to its high stability in biological media $(<9 \%$ degradation in 72 hours) and (iii) slow NO release in biological media ( $\approx 2$ hours for $90 \%$ release). The prospective application of MIP-177 is demonstrated through NO-driven control of mitochondrial respiration in cells and stimulation of cell migration, paving the way for the design of new NO delivery systems for wound healing therapy.
\end{abstract}

\section{Introduction}

Nitric oxide (NO) plays an important role in signaling pathways in human physiological and pathological processes, garnering considerable interest in its potential as a therapeutic agent. In particular, exogenous delivery of NO has been widely demonstrated to have a therapeutic effect in wound healing ${ }^{[1,2]}$ through its promotion of vascular permeability ${ }^{[3]}$ and killing of pathogens. ${ }^{[4]}$ It is also currently considered as an anti-oncogenic agent. ${ }^{[5]}$ However, due to the high toxicity and short half-life, delivery of NO to the target sites in a controlled manner remains a great challenge. For this purpose, ordered nanoporous materials have recently been proposed as carriers for the controlled local delivery of pure NO that homogeneous molecular donors fail to achieve ${ }^{[6]}$ These nanoporous delivery materials, including zeolites, ${ }^{[7,8]}$ titanosilicates, ${ }^{[9,10]}$ and metal-organic frameworks (MOFs) ${ }^{[11-14]}$ have demonstrated considerable NO storage and release capacity. Some of these materials can even form metal-NO coordination bonds pointing towards the pores, allowing the adsorption of large amounts of NO with a better controlled release. Another approach, using porous coordination polymers (PCPs) functionalized with $\mathrm{N}$-nitrosamine functional groups or incorporating nitro-containing imidazolate ligands with poor reactivity into the PCP frameworks, demonstrated efficient and controlled release of NO using light radiation. ${ }^{[15,16]}$

However, the toxicity and stability issues of all these nanomaterials, as well as the biological impact of the NO encapsulation/release have scarcely been explored so far. ${ }^{[11,12,17,18]}$ Moreover, the design of the ideal nanoporous NO carrier is still a very demanding challenge since the material needs to be biocompatible and stable in aqueous biological media (i.e., no signs of toxicity at therapeutic concentrations or release of metals when in contact with cells for several days), feature strong NO storage capacity (currently best materials are capable of storing 1-7 $\mu \mathrm{mol} \mathrm{NO}$ $\left.\mathrm{mg}^{-1}\right),{ }^{[12,13,19]}$ and slow NO release in biological media during several hours since the biological activity and toxicity of this molecule is concentration-dependent. ${ }^{[2-22]}$ To our knowledge, such a critical combination of features has not been achieved so far. The development of new solid NO donors is thus required to overcome the limitations of the state-of-the-art molecular donors, that are, inadequate long-term delivery,

R. V. Pinto, ${ }^{[+]}$Prof. J. Pires, Prof. F. Antunes

Centro de Química e Bioquímica e CQE, Faculdade de Ciências,

Universidade de Lisboa

1749-016 Lisboa (Portugal)

Dr. A. Vimont, Prof. G. Clet, Prof. M. Daturi

Normandie Univ, ENSICAEN, UNICAEN, CNRS, Laboratoire Catalyse et Spectrochimie

14000 Caen (France)

Dr. S. Wang ${ }^{[+]}$

Current address: Hefei National Laboratory for Physical Sciences at the Microscale, University of Science and Technology of China Hefei (China)

$\left.{ }^{+}\right]$These authors contributed equally to this work.

(2) Supporting information and the ORCID identification number(s) for (iD the author(s) of this article can be found under: https://doi.org/10.1002/anie.201913135. 
instability during storage and issues associated to systemic circulation of potentially toxic nanovectors. ${ }^{[6,23]}$ To that end, biocompatible MOFs with coordinatively unsaturated sites (CUSs) in their porous structures could provide a potential solution.

MOFs are one of the newest classes of porous crystalline materials that have recently been explored for a wide range of medical applications, ${ }^{[24-27]}$ with a great potential for the encapsulation and release of drugs. ${ }^{[25]}$ Nevertheless, biocompatible MOFs considered so far have exhibited major drawbacks, such as limited structural stability in biological media strongly affecting the NO release performance, also leading to the release of metals into the media which may, in some cases, induce toxicity ${ }^{[13,26,28]}$ Indeed, the MOF structure has to be sufficiently stable, particularly if an effective release over several hours is targeted as, for example, in wound healing applications.

Herein, we have considered a very recently reported titanium carboxylate MOF, denoted MIP-177 (Figure 1), in its low temperature, formate-free form (MIP stands for the materials of the Institute of Porous Materials of Paris), that fulfils the requirements for an ideal NO delivery system.

reference required for MIP-177? $\mathbf{\square}$ We here demonstrate that this non-toxic material is highly stable in biological media and exhibits a porous structure featuring titanium metal sites on its inorganic building unit in an ideal position to coordinate NO by the uncommon in situ formation of complexed nitrites. The performance of this new donor in biological systems has been analyzed by testing different $\mathrm{NO}$ concentration ranges until the desired effect is observed, that is, the stimulation of cell migration. This is a fundamental process in wound healing and, to the best of our knowledge, is being explored for the first time with this class of NO donors. This MOF material, which offers the ability to exercise control over certain basic biological processes, represents a step forward in the development of new strategies for therapeutic uses such as the acceleration of wound healing.

\section{Results and Discussion}

\section{Synthesis and Characterization of the Ti-MOF}

The use of active Ti open metal sites was reported to be attractive for NO coordination and delivery in the microporous titanosilicate ETS-4. ${ }^{[9]}$ However, harsh preparation conditions required on a small scale and limited accessible porosity resulting in restricted accessibility to the Ti metal sites are clear limitations of this solid. Moreover, ETS-4 exhibits fast release of $\mathrm{NO}$ upon contact with biological media due to its high hydrophilicity, as will be shown here. To address those limitations, MIP-177 in its formate-free form with a chemical formula $\mathrm{Ti}_{12} \mathrm{O}_{15}(\operatorname{mdip})_{3}(\mathrm{OH})_{6}\left(\mathrm{H}_{2} \mathrm{O}\right)_{6}(\mathrm{mdip}=$ $3,3^{\prime}, 5,5^{\prime}$-tetracarboxydiphenylmethane), has been selected as NO storage and release candidate based on its following features: (i) robust porosity with adequate adsorption sites to enable large NO uptake and release. As shown in Figure 1, MIP-177 comprises nanosized one-dimensional (1D) channels with a free diameter of $1.1 \mathrm{~nm}$ running along the $c$-axis. Each $\mathrm{Ti}_{12} \mathrm{O}_{15}$ secondary building unit (SBU) possesses three pairs of terminal $\mathrm{OH} / \mathrm{H}_{2} \mathrm{O}$ species facing the pore, which can be removed thermally to generate bridged $\mathrm{Ti}-\mathrm{OH}-\mathrm{Ti}$ as evidenced by coupling density functional theory (DFT) calculations and in situ infrared spectroscopy (FTIR) analysis (see Supporting Information); (ii) excellent hydrolytic and chemical stability under various conditions as reported previously ${ }^{[29]}$ and more specifically in biological media. This is of great importance to avoid any toxicity and performance fluctuation during application in real biological systems; and (iii) an easy and environmentally friendly preparation for future scale-up applications. MIP-177 particles are of elongated shape with uniform sizes between 0.1 and $0.2 \mu \mathrm{m}$ in length that form agglomerates of about $1 \mu \mathrm{m}$ (Figure S21, Supporting Information), which are biocompatible as shown below.
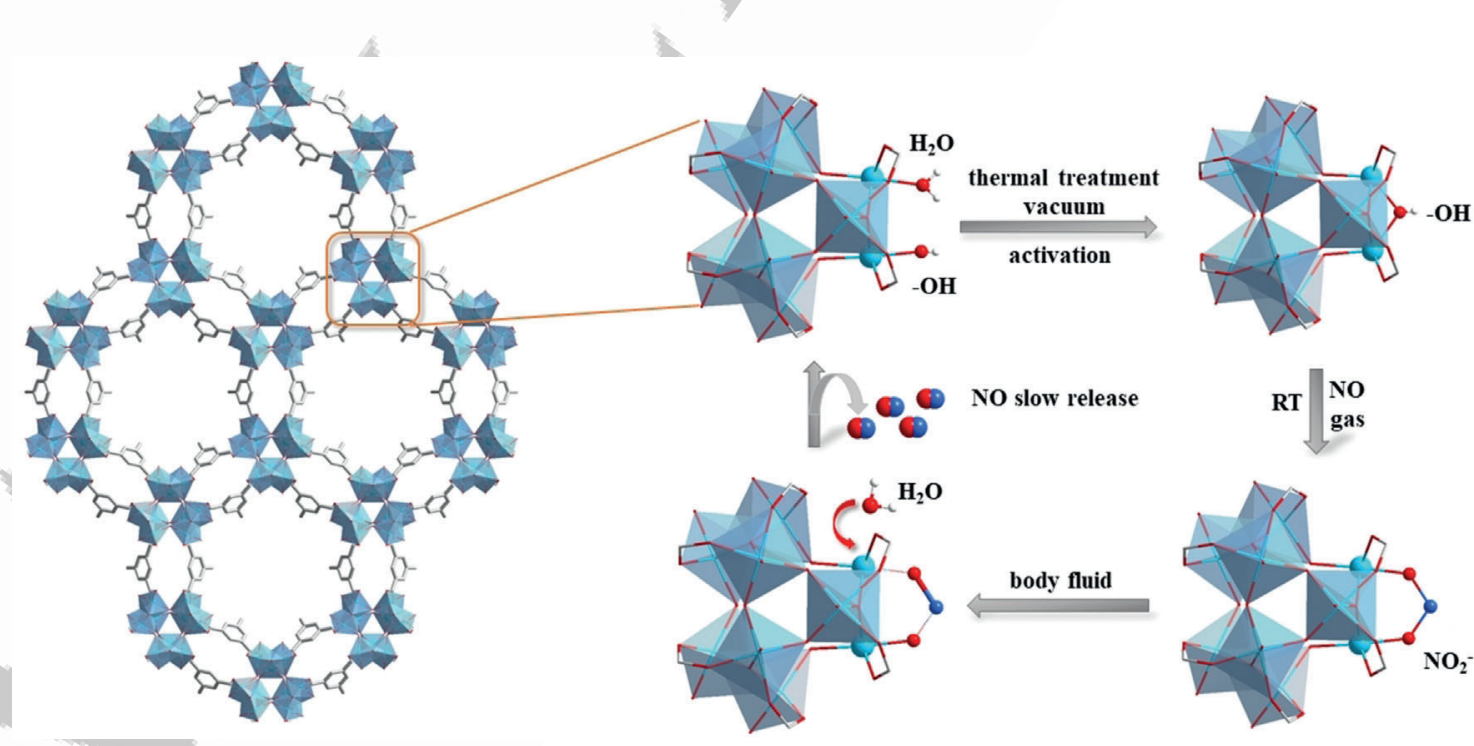

Figure 1. MIP-177 microporosity and binding/release mechanism: viewed along the $c$-axis (left), adsorption and controlled release cycle of NO under the tested conditions (right) is shown. 
Computational Prediction of Interactions Between NO and MIP177

DFT calculations were first performed for the "empty" MOF material; the formate groups connecting two Ti sites were replaced by one terminal $\mathrm{OH}$ bound to one Ti site, leaving the second $\mathrm{Ti}$ site uncoordinated to mimic a dehydrated state. The DFT-geometry optimization of such a configuration led to the formation of bridging $\mathrm{OH}$ groups bonded to both Ti sites (Figure S1), which is in agreement with FTIR observations. These $\mathrm{OH}$ groups are the preferential interacting site for $\mathrm{NO}$ with a $\mathrm{H}(\mathrm{OH})-\mathrm{O}(\mathrm{NO})$ distance of $2.9 \AA$ (Figure S2a), associated with a high binding energy of $72 \mathrm{~kJ} \mathrm{~mol}^{-1}$ which however remains lower than the value previously reported for the adsorption of $\mathrm{NO}$ on the Ni CUSs in Ni-CPO-27 $\left(90 \mathrm{~kJ} \mathrm{~mol}^{-1}\right){ }^{[30]}$ We have evidenced that even when $\mathrm{OH}$ is forcibly bound in the terminal position to one $\mathrm{Ti}$ site, leaving the neighbouring Ti site uncoordinated, NO does not interact strongly with the Ti CUS (Figure S2a). Furthermore, the comparison of the total energies of both configurations (Figure S2a and S2b) indicates that the interaction of $\mathrm{NO}$ with the $\mathrm{OH}$ bridge is highly favoured. Therefore, as we will demonstrate in the subsequent sections, the bridging $\mathrm{OH}$ group will react with $\mathrm{NO}$ to produce bridging or chelating nitrites (Figure 1).

\section{Therapeutic NO Release}

NO adsorption on MIP-177 reveals a maximum storage capacity of $9 \%(\mathrm{w} / \mathrm{w})$ (total of $3 \mu \mathrm{mol} \mathrm{mg}^{-1}$ solid) after 27 hours (Figure 3a), with fast loading kinetics, reaching $6 \%(\mathrm{w} / \mathrm{w})$ in the first 5 hours. Considering the theoretical number of binding sites available in MIP-177 and the in situ formation of nitrite species to generate $\mathrm{Ti}_{12} \mathrm{O}_{15}(\text { mdip })_{3}(\mathrm{NO}(\mathrm{OH}))_{6}$ (Figure 1 and in situ IR discussion below), the maximum expected loading capacity is about $9.6 \mathrm{wt} \%$ (see Supporting Information for details), which is close to the observed maximum storage capacity. This indicates that MIP-177 is among the best MOFs so far reported in the literature $\left(0.8-7 \mu \mathrm{mol} \mathrm{mg}^{-1}\right)$ for NO storage. ${ }^{[12,13,31,32]}$

The kinetic desorption curve in Figure $3 b$ ) shows quick NO release in the first 2 hours, reaching an equilibrium after 20 hours. NO amounts released from MIP-177 $\left(1.7 \mu \mathrm{molmg} \mathrm{m}^{-1}\right.$; about $55 \%$ of the loaded amount) exceed those of previously reported porous materials. For example, zeolites, clay-based materials and titanosilicates such as ETS10 , ETS-4 and some modified formulae all present values $\leq 1.7 \mu \mathrm{mol} \mathrm{mg}^{-1} \cdot{ }^{[9,10,33-36]}$ Furthermore, the release performance of MIP-177 is higher than reported for most MOFs, only CPO-27 exceeds the value of MIP-177..$^{[12,13,19]}$ Remarkably, $45 \%$ of the loaded NO is retained in the material even under high vacuum, which is in agreement with the formation of strongly-bound nitrite species (Figure 1 and 2). The retained NO is released when the material is introduced to biological media (demonstrated below).

NO adsorption and desorption were also monitored by FTIR (Figure 2). Several species were formed over a 24-hour adsorption period, which subsequently exhibited different desorption behaviors. Shortly after NO introduction, a new band quickly developed at $1850 \mathrm{~cm}^{-1}$ and was assigned to weakly adsorbed NO, as previously reported for NO adsorption on MIL-88(Fe). ${ }^{[37]}$ This band did not evolve significantly after 3 hours and the weak interaction with the pore walls was confirmed by its disappearance upon evacuation at room temperature. At the same time, a band at $1210 \mathrm{~cm}^{-1}$ appeared reaching it maximum after about 3 hours, at which point it started decreasing (Figure S5). This spectral range is typical of
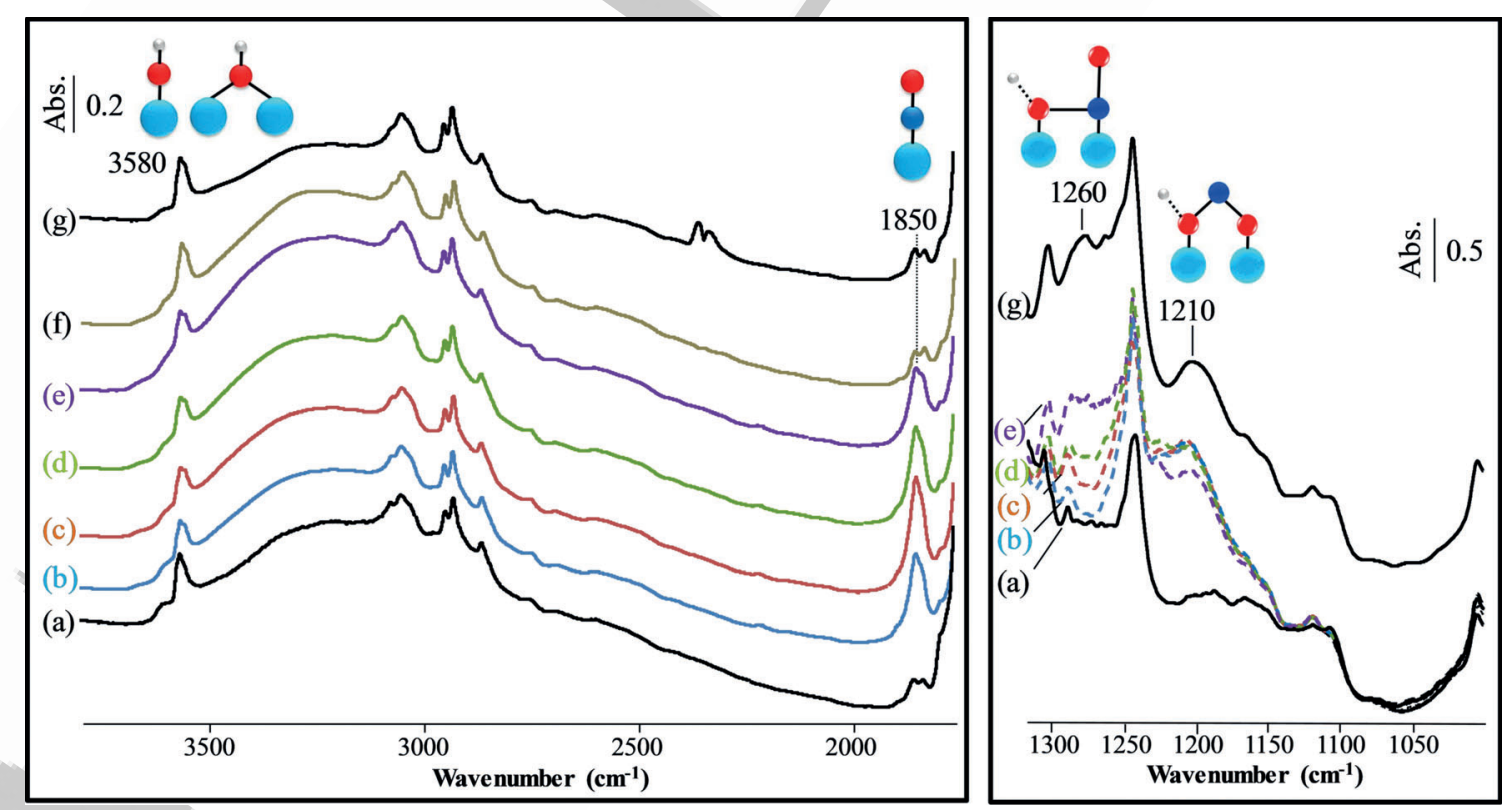

Figure 2. Infrared absorption spectra of MIP-177 recorded after thermal treatment at $120^{\circ} \mathrm{C}$ (a) and after introduction of NO at room temperature $\left(p_{\mathrm{NO}}=80 \mathrm{kPa}\right)$ at exposure times of $20 \mathrm{~min}(\mathrm{~b}), 75 \mathrm{~min}(\mathrm{c}), 195 \mathrm{~min}(\mathrm{~d}), 20$ hours (e), followed by outgassing at room temperature for $20 \mathrm{~min}(\mathrm{f})$ and at $120^{\circ} \mathrm{C}$ for 2 hours (g). Adsorbed species representation: $\mathrm{O}=\mathrm{Ti}, \mathrm{O}=\mathrm{N}, \mathrm{O}=\mathrm{O}, \mathrm{O}=\mathrm{H}$.

\section{These are not the final page numbers!}


bridging nitrites or nitrates. ${ }^{[38]}$ The possibility of nitrate formation can be eliminated as the parallel development of characteristic bands around $1020 \mathrm{~cm}^{-1}$ is not observed in the spectrum. Therefore, the band at $1210 \mathrm{~cm}^{-1}$ can be confidently assigned to bridging nitrites $(\mathrm{Ti}-\mathrm{O})_{2}=\mathrm{N}$. These modifications are associated with an initial decrease of the band at $3580 \mathrm{~cm}^{-1}$ and the development of a broad band around $3300 \mathrm{~cm}^{-1}$ implying the involvement of the hydroxyl species and its perturbation (or even shift, in the form of adsorbed water molecules) in this first step, to allow nitrite formation. Adsorption proceeds through a second step since another band develops at $1260 \mathrm{~cm}^{-1}$, which was assigned to chelating nitrites. ${ }^{[38,39]}$ Unlike the previous bands, this one slowly increased over the first 200 minutes, and continued growing steadily for up to 24 hours. A slight development of the band at ca. $3300 \mathrm{~cm}^{-1}$ was also observed, while the bands at 1210 and $3580 \mathrm{~cm}^{-1}$ were progressively decreasing in parallel, with a trend similar to that of the band at $1260 \mathrm{~cm}^{-1}$ (Figure S5). This is a clear indication that chelating nitrites form at the expense of some bridging nitrites and provoke the removal of weakly coordinated hydroxyls, as schematized in Figure S6. Upon evacuation, bands at both 1210 and $1260 \mathrm{~cm}^{-1}$ persisted, conversely to the band at $1850 \mathrm{~cm}^{-1}$, evidencing their stability. Therefore, we conclude that NO adsorption induces the formation of both weakly adsorbed species and stable nitrites.
This is in excellent agreement with the release of only ca. $55 \%$ of the loaded NO after outgassing, leaving the remaining $45 \%$ of the loaded NO in the MOF as very stable nitrites bonded to a pair of Ti sites.

To further investigate chelating and bridging nitrite/ nitrate adsorptions, DFT calculations were performed

ak? (see Figure S3 and S4). In case of the nitrite adsorption, the DFT calculations showed that chelating and bridging species may coexist since the two geometries converge towards a very similar energy. The configuration corresponding to the bridging nitrate species shows a substantially higher stability than that of the chelating nitrate species, with a relatively high energy difference of $17.1 \mathrm{~kJ} \mathrm{~mol}^{-1}$. Finally, regarding the formation of the nitrite/nitrate species, the energy comparison between corresponding configurations (see discussion in the Supporting Information) indicated that the nitrite adsorption is much more favored. We estimated a binding energy of $134.1 \mathrm{~kJ} \mathrm{~mol}^{-1}$ for the nitrites on the Ti sites (Supporting Information) which is significantly higher than the values previously reported for Ni-CPO-27. ${ }^{[30]}$ Therefore, the DFT simulations and the IR measurements concur in terms of the presence of stable nitrite species chemisorbed on the Ti sites, which support the adsorption results (Figures $3 \mathrm{a}$ and $b$ ).
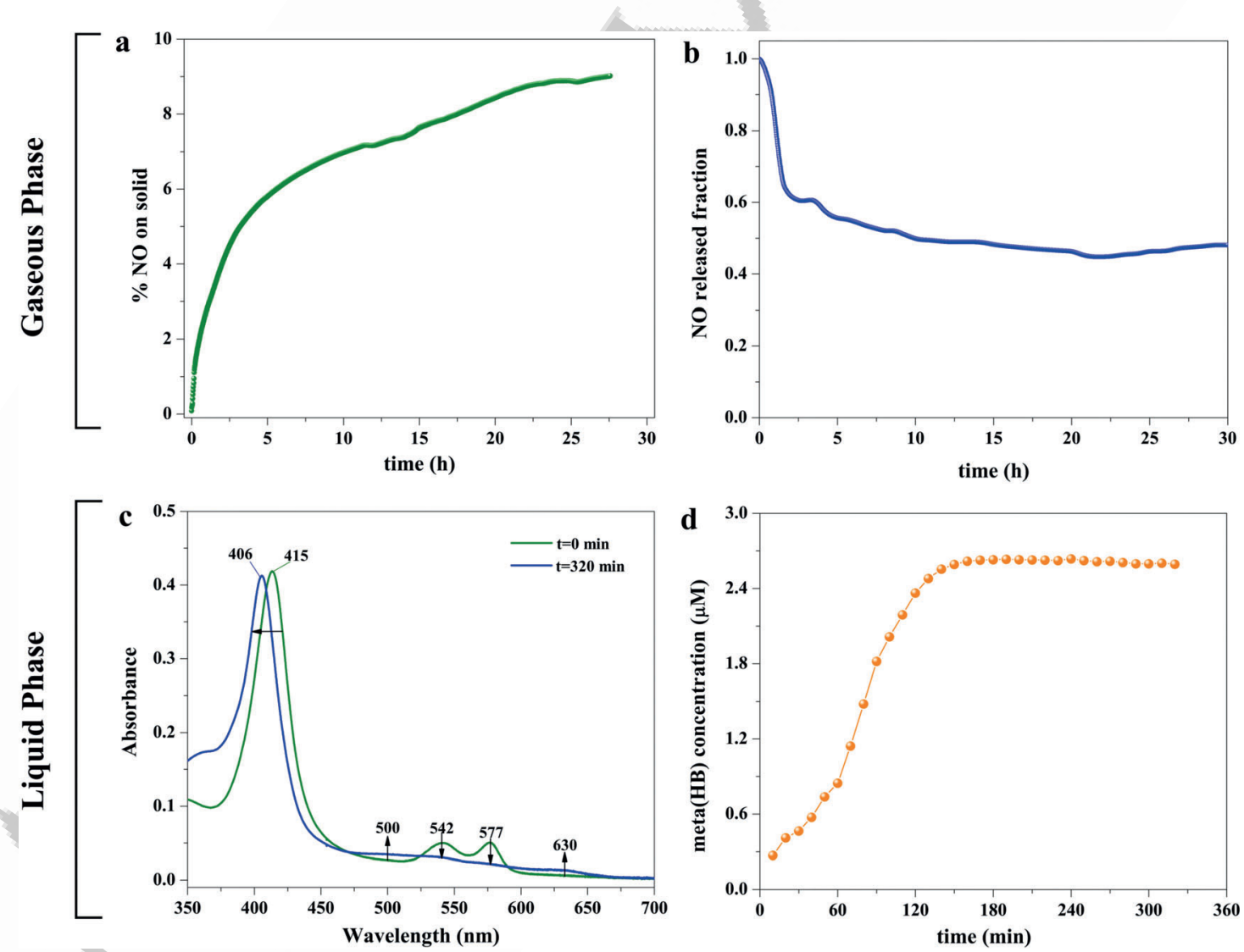

Figure 3. NO adsorption/release capacities in gas and liquid phase. a, b) NO kinetic profiles performed on a gravimetric apparatus, at $25^{\circ} \mathrm{C}$ : a) represents $\mathrm{NO}$ adsorption performed at $80 \mathrm{kPa}$ and b) $\mathrm{NO}$ release under vacuum of the studied material; $\mathrm{c}$, d) NO release in liquid phase using the oxyhemoglobin assay, performed at $25^{\circ} \mathrm{C}$ using phosphate buffer solution: c) comparison between the initial UV/Vis spectrum of the oxyhemoglobin solution with the final UV/Vis spectrum (after $3 \mathrm{~h}$ ) and d) NO kinetic release profile from the MIP-177 loaded with NO, in oxyhemoglobin solution. 
These experimental and computational results shed light on a new conceptual mechanism of NO storage/release using porous solids. Until now, only MOFs bearing single unsaturated metal sites (CUS), such as CPO-27/MOF-74 or MIL$100(\mathrm{Fe})$, have been considered for NO delivery applications. The adsorption/release mechanism relied on direct NO coordination onto these metal sites. Herein, MIP-177, with its unusual building unit involving two neighboring Ti sites, allows the in situ formation of bridging or chelating nitrite species upon adsorption of NO, resulting in a species more strongly bound to the framework than a single-bonded NO molecule to a typical CUS.

Previously, NO-loaded MOFs were mostly studied for antimicrobial or anti-thrombogenic applications, in most cases not in direct contact with biological fluids. These media are more challenging for the controlled release of NO due the faster exchange between $\mathrm{NO}$ and liquid water as well as the presence of phosphates that rapidly degrade most metal carboxylate MOFs. Given the enhanced stability of NO species bound to MIP-177, we decided to consider this new NO donor for biological applications. NO release kinetics were thus further evaluated in the liquid phase, utilizing several complementary methods. Using an oxyhemoglobin assay $^{[40]}$ (Figure $3 \mathrm{c}$ and $\mathrm{d}$ ), we revealed the selective reaction of NO with oxyhemoglobin when NO-loaded MIP-177 was placed in contact with the solution. Changes in the UV/vis spectrum over time (Figure $3 \mathrm{c}$ ) were attributed to the transformation of oxyhemoglobin into methemoglobin, demonstrating NO release (this method is not sensitive to nitrite or nitrate species). The NO release kinetics (Figure $3 \mathrm{~d}$ ) show a stabilization in the concentration after about 150 minutes, indicating complete release or complete oxyhemoglobin consumption. Nevertheless, the initial slope shows a slow release of NO. In another test, the loaded material was introduced to RPMI-1640 cell culture medium at ambient conditions. NO release was followed by a direct electrochemical measurement (NO selective electrode). For a MIP177 concentration of $450 \mu \mathrm{gLL}^{-1}$, the release reached a maximum of $100 \mathrm{~nm}$ (concentration decreases afterwards as NO can react with $\mathrm{O}_{2}$ in the medium) with a very attractive slow kinetic profile, showing a controlled release over approximately 2 hours, which has not been observed for any other porous material before (Figure S7 and discussion/comparison with other materials). Such a slow release is likely related to the formation of bridging or chelating nitrites inside the MIP177 , which are decomposing slowly through a reaction with water and rehydration of the Ti metal sites, providing a stable concentration of $\mathrm{NO}$ in the liquid phase ${ }^{[41,42]}$ (NB: ion exchange with phosphates is less likely, since no significant phosphate concentration was found in MIP-177 after some days in phosphate solution). A Griess assay was also performed for 2 hours using different concentrations of NOloaded MIP-177 under in vitro conditions (Figure S8). The overall results obtained for NO release in the liquid phase demonstrated that MIP-177 can release active NO with particularly slow release kinetics (an extended discussion of this point is presented in the Supporting Information). These unique properties can be explained by the different nature of NO bonding inside the pores of MIP-177 compared to that of other MOFs. Indeed, this type of binding is more similar to NO chemisorption in some polymers and dendrimers to form RSNOs or NONOates. ${ }^{[20]}$ However, MIP-177 shows the advantage of a larger storage capacity, affords more control over the molecular structure of the NO vehicle, has very low toxicity and provides additional optionality via its large pores which may be used to store additional drug molecules for combined synergic delivery and biological effect. ${ }^{[20,26]}$

A toxicity assessment using two different cell lines (HeLa and primary human epidermal keratinocytes-HEKn) was performed with MIP-177 at $180 \mu \mathrm{g} \mathrm{mL}^{-1}$ and $450 \mu \mathrm{g} \mathrm{mL}^{-1}$. HEKn was chosen to assess the potential application of this new material in wound-healing, while HeLa was selected because it is a widely used cell line for which a significant number of cytotoxicity studies can be found in the literature. No significant toxicity was observed, even at high concentrations of the solid (Figure S9). Only in the case of HEKn, a very low toxicity was found after 24 hours, and after 72 hours a decrease in the viability of the cells $(71 \pm 8 \%$ at $450 \mu \mathrm{g} \mathrm{mL}^{-1}$ ) was observed. However, as shown below, NO loaded MIP-177 will produce a positive effect on cell migration at concentrations of $90 \mu \mathrm{g} \mathrm{mL}^{-1}$, for which no toxicity was found. Compared with previous work, where all porous materials studied for the same purpose presented at least $20 \%$ toxicity with HeLa cells after 72 hours, ${ }^{[3-35,43]}$ no toxicity was observed in the present case with HeLa cells (a comparison with other MOFs is presented in the Supporting Information).

MIP-177 stability, another critical requirement, ${ }^{[45]}$ was evaluated under biological conditions. The $\mathrm{Ti}^{4+}$ concentration in the liquid medium after 72 hours was 0.5 and $1.1 \mu \mathrm{g} \mathrm{mL}^{-1}$ in HeLa and HEKn cell media, respectively, indicating a merely residual degradation of the material (about $9 \%$ in the worst case). The low degradation was confirmed by PXRD (Figure S12a) and by SEM images (Figure S12b). This is in contrast with the MOFs tested so far, which have not been stable enough in biological media (e.g., iron carboxylates MIL-88's, MIL-100 and MIL-127 or metal(II) hydroxycarboxylate $\mathrm{CPO}-27(\mathrm{Mg}, \mathrm{Zn})$ and/or MOFs containing very toxic metals, such as $\mathrm{Cu}$ carboxylates or $\mathrm{CPO}-27(\mathrm{Cu}, \mathrm{Mn}, \mathrm{Ni}$ or $\mathrm{Co})$ ), hindering potential future therapeutic applications, ${ }^{[12,14,32]}$ particularly topical NO delivery to wounds. For instance, biocompatible iron MOFs (Fe-MIL-100 and FeMIL-127) ${ }^{[32]}$ were submitted for the same stability tests and showed high concentrations of iron in both media after 72 hours (between 1.39 to $7.31 \mu \mathrm{g} \mathrm{mL}^{-1}$, Table S1). This instability may affect the controlled release of $\mathrm{NO}$ in biological media (a spike in released NO is observed in the first minutes upon contact with moisture, which renders those MOFs unsuitable for medical applications). ${ }^{[32]}$ These results underline the exceptionally low toxicity and excellent stability of MIP-177 which are highly attractive qualities for topical NO delivery applications.

To prove the efficiency of this new material as a viable vehicle for the delivery of NO in biological applications, a relationship between the amount of NO released and the effect observed in biological systems must be clearly demonstrated. So far, it has only been demonstrated in the literature that $\mathrm{NO}$ released from porous materials can trigger some 
positive responses in artery relaxation tests ${ }^{[12,46]}$ and in the inhibition of platelet aggregation, but the effect of the material concentration on the response has not been reported yetם $\square$ ok? ${ }^{[7]}$ Recently a NO-releasing zeolite was studied for wound healing. However, it showed a reduced effect, which is associated with the high toxicity of the NOloaded material, likely due to its fast NO release (see Supporting Information for further discussion). ${ }^{[18]}$ In this case, we have chosen two relevant cellular processes that can be regulated by $\mathrm{NO}^{[47,48]}$ to demonstrate our ability to control them with NO-loaded MIP-177: (1) mitochondrial respiration, which was evaluated by measuring the oxygen consumption of cells, and (2) the wound healing process simulated using a cell migration assay.

Oxygen consumption was monitored in a respiration buffer before and after NO-loaded MIP-177 was added $\left(450 \mu \mathrm{g} \mathrm{mL}^{-1}\right)$. A reversible inhibition of mitochondrial respiration by the released NO was observed (Figure $4 \mathrm{~b}$ ), reaching the highest inhibition rate of $78.5 \%$, after which the respiration recovered to $70 \%$ of the initial value after 55 minutes. Results from $\mathrm{NO}$ and $\mathrm{O}_{2}$ sensors (Figure $4 \mathrm{a}$ and $\mathrm{b}$ ) were in agreement, showing that oxygen consumption was inhibited when the material was introduced and NO release

a

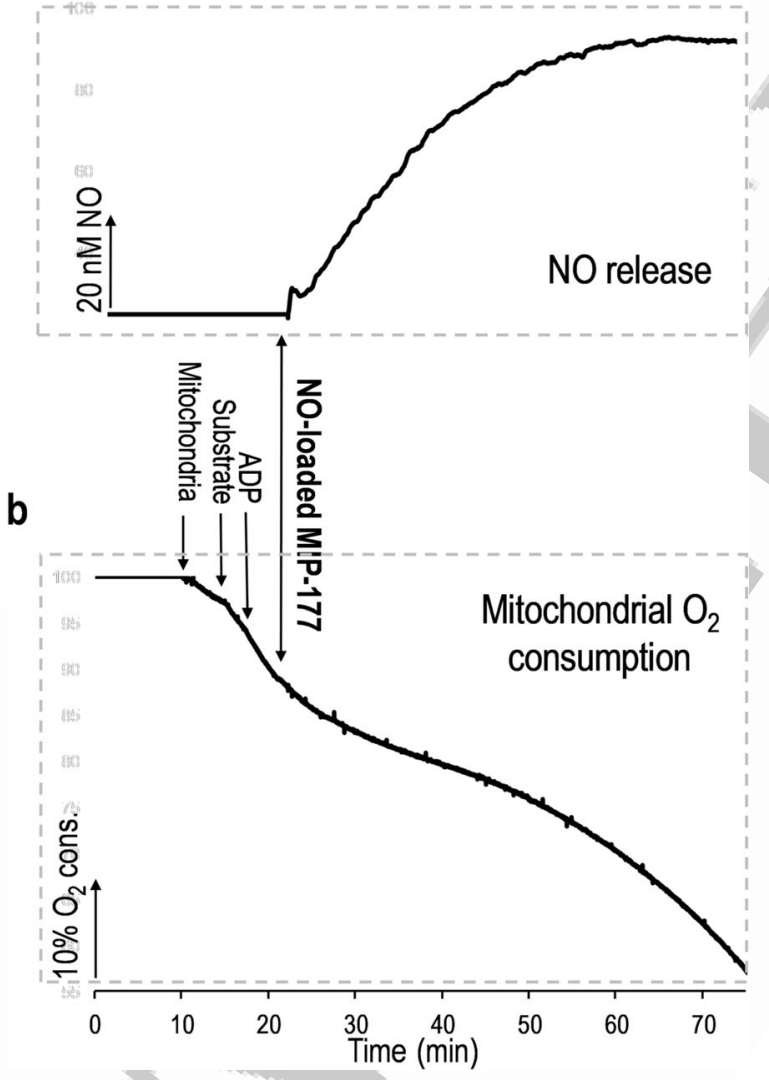

Figure 4. Effects on mitochondrial respiration in the presence of NOloaded MIP-177. a) Release profile obtained from the NO-sensitive electrode using a MIP-177 concentration of $450 \mu \mathrm{g} \mathrm{mL}^{-1}$ in supplemented culture medium (RPMI-1640); b) Mitochondrial respiration assay using digitonin-permeabilized HeLa cells ( $1.25 \mathrm{mg}$ of protein) with $450 \mu \mathrm{g} \mathrm{mL}^{-1}$ of NO-loaded MIP-177, tested by measuring the $\mathrm{O}_{2}$ consumption with a $\mathrm{O}_{2}$-sensitive electrode. was started, and increasing further (Figure 4b) when the rate of NO release started to slow down (Figure $4 \mathrm{a}$ ). At lower concentrations (Figure S13 and S14), the released NO causes a lower inhibition of the mitochondrial respiration rate ( $\approx 18 \%$ at $180 \mu \mathrm{g} \mathrm{mL}^{-1}$ and no inhibition at $90 \mu \mathrm{gLL}^{-1}$ ). The high dependence of the mitochondrial respiration on NO concentration is demonstrated, proving that the NO released from MIP-177 indeed influences oxygen consumption and that it can be controlled by varying the concentration of material. These results are in agreement with the literature: low nanomolar concentrations of NO specifically inhibit cytochrome oxidase (the terminal complex of the mitochondrial respiratory chain) in competition with oxygen, and this inhibition is reversed when NO is removed. ${ }^{[47,49]}$

It is reasonable to assume that this new NO donor could promote cell migration and wound closure and be a suitable therapeutic alternative in skin reparatory processes. To confirm such a possibility, the cell response to exposure with different concentrations of NO-loaded MIP-177 was evaluated using migration assays. After treating the cells at a concentration of $90 \mu \mathrm{g} \mathrm{mL}^{-1}$ (Figure $5 \mathrm{a}$ ), an initial increase in the migration rate was observed $(5.5 \pm 1.3 \%)$ after 6 hours, achieving an improvement in wound closure of $8.4 \pm 1.4 \%$ after 24 hours. These results suggest that a sustained effect could be achieved by renewal of the supply of NO-loaded MIP-177 to the medium every day, which is convenient for therapeutic purposes. Microscopic images (Figure 5), which show pre- and post-migration period cultures exposed to NOloaded and unloaded (control) MIP-177, confirm the increased rate of wound closure with increased released NO. Some MIP-177 particle aggregates can be seen on the images, scattered outside the cells. Additional images that elucidate the particles in the cell cultures are shown in Figure S15). At higher concentrations of NO-loaded MIP-177 $\left(180 \mu \mathrm{g} \mathrm{mL}^{-1}\right.$, Figure S16), no significant difference in the migration rate was observed. Since NO effects are extremely concentration dependent, perhaps the NO amount released from MIP-177 at $180 \mu \mathrm{g} \mathrm{mL}^{-1}$ was not suitable for this application, possibly due to an excessive supply of NO to the media (about the double amount of NO compared to $90 \mu \mathrm{g} \mathrm{mL}^{-1}$ sample shown in Figure S8). To the best of our knowledge, this is the first time that NO released from MOFs has been studied as an alternative for skin reparatory therapy. So far, only homogeneous NO donors have been considered for this therapeutic application. Zhan et al. ${ }^{[50]}$ reported that migration of HaCaT cells may be promoted either by Sodium nitroprusside

name ok? (SNP), spermine NONOate, or $S$-nitroso- $N$ acetylpenicillamine $\square$ name ok? $\mathbf{\square}$ (SNAP) as homogeneous donor in a dose-dependent manner. $10 \mu \mathrm{M} \mathrm{SNP,} 10 \mu \mathrm{M}$ spermine NONOate and $100 \mu \mathrm{M}$ SNAP were found to be the optimal concentrations to induce cell migration, promoting an increase of ca. $1-10 \%$ in the first 6 hours, in the same range as found here with MIP-177. Higher amounts presented inhibitory and cytotoxic effects were observed for the highest concentration $(1000 \mu \mathrm{M})$. Other studies have also reported that exogenous NO supply using NO donors induces enhanced cell proliferation and migration in a concentrationdependent manner. ${ }^{[51-53]}$ However, current homogeneous donors can only provide non-targeted NO release since they 

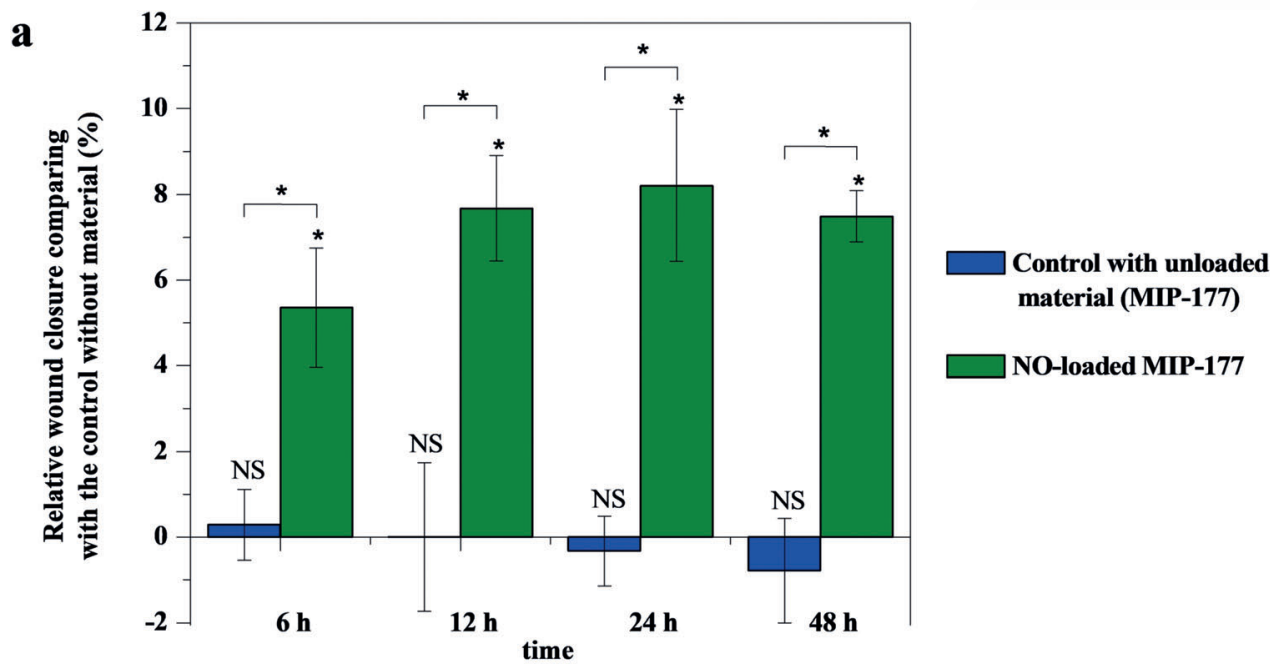

b
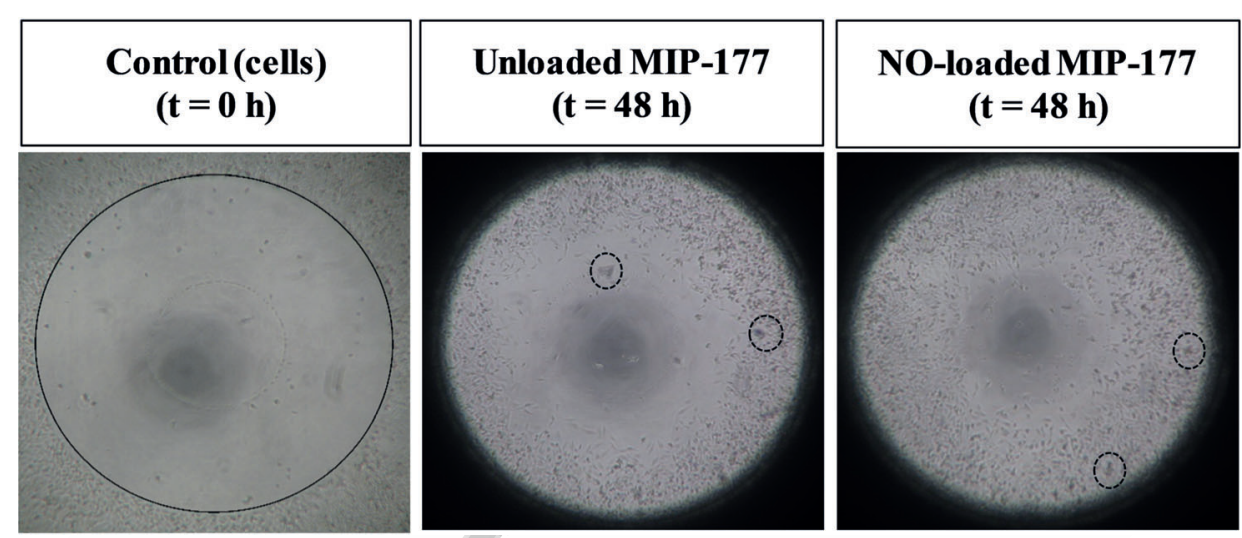

Figure 5. Effects of released NO on cell migration and wound closure. a) Cell migration data obtained by treating HeLa cells with MIP-177 as the NO donor material at a concentration of $90 \mu \mathrm{g} \mathrm{m}^{-1}$. b) Microscope images captured during the cell migration assay. The first image on the left, captured without a detection mask in place shows the cells (control) in the pre-migration period ( 0 hours). The middle picture, captured with a detection mask, shows the migration of the control cells treated with unloaded MIP-177 post-migration (48 hours). The first image on the right, captured with a detection mask, shows the cells treated with NO-loaded MIP-177 post-migration (48 hours) (90 $\left.\mu \mathrm{g} \mathrm{mL}^{-1}\right)$. As shown in the images of cells post-migration, the presence of the scattered MIP-177 particles between the cells is evident (some examples outlined in black circles). Data in a) represents the average percentage migration \pm SD from three independent experiments, each performed in quadruplicate. Both conditions (cells treated with unloaded and NO-loaded material) are relative to the control (cells without material), that is, the difference in the percentage of wound closure between the tested condition and the control. Data were compared using the unpaired student's t-test to assess significance. $* P<0.05$. Additional details in the Supporting Information.

can disseminate throughout tissues and body, which may affect their therapeutic efficiency and cause harsh side effects in vivo ${ }^{[6,54]}$ Moreover, the decomposition products formed by the donors after releasing NO could be toxic and affect the surrounding tissues. ${ }^{[5]}$ In the case of MIP-177, a controlled release of pure NO is guaranteed, which, combined with its excellent stability and biocompatibility, are novel characteristics that support the use of MIP-177 as a suitable alternative in wound healing applications.

To satisfy specific medical requirements, the ability to adjust NO release kinetics and amounts of NO released from donors is highly desirable. In nanoporous carriers, these are dependent on pore structure and surface chemistry of the NO donor. ${ }^{[6]}$ The properties can vary drastically from one porous material to another. Thus, fine tuning of the same MOF structure would be an interesting approach to achieve specific modifications. To explore this concept, the same structure was prepared, but with a partial isomorphic substitution of the $\mathrm{Ti}^{\mathrm{IV}}$ metal centres with $\mathrm{Fe}^{\mathrm{III}}$ ( $15 \%$ of Ti replaced by $\mathrm{Fe}$ ), giving a new material with the same structure and two types of metal centres. Indeed, this preliminary study showed that this led to a slight increase of the stored amount of NO and, most importantly, that this approach could be further exploited to fine tune NO release capacity and kinetics (Figure S17).

\section{Conclusion}

The robust titanium carboxylate MOF MIP-177 features a new mechanism for $\mathrm{NO}$ adsorption/release through the formation of complexed nitrites on its inorganic building unit. MIP-177 furthermore shows a combination of excellent biocompatibility, particularly at therapeutically relevant concentrations, and unprecedented stability in biological media, 
which has led to a remarkably slow NO release in biological fluids. This facilitates control over biological responses that exhibit a dependence on the concentration of NO. These conclusions provide a step forward in the development of advanced nanoporous materials for NO delivery for use in wound healing therapy. MIP-177 can indeed be considered as a new component in the development of NO delivery platforms, to be potentially deployed in combination with polymers, gels and ointments for future practical clinical applications.

\section{Acknowledgements}

The work was financed by Fundação para a Ciência e a Tecnologia (FCT) through projects IF/00993/2012/CP0172/ CT0013 and PTDC/MED-QUI/28721/2017. This work was developed in the scope of the Projects UID/MULTI/00612/ 2019 (CQB), UID/ECI/04028/2019, UIDB/04028/2020 (CERENA) and Programa Investigador FCT, financed by national funds through the FCT/MEC and co-financed by FEDER under the PT2020 Partnership Agreement. Hazelle Wang is acknowledged for proof reading of the manuscript.

\section{Conflict of interest}

The authors declare no conflict of interest.

Keywords: IR spectroscopy - metal-organic frameworks . modelling $\cdot$ nitric oxide $\cdot$ wound healing

[1] Y. Kang, J. Kim, Y. M. Lee, S. Im, H. Park, W. J. Kim, J Controlled Release 2015, 220, 624-630.

[2] F. S. Schanuel, K. S. R. Santos, A. Monte-Alto-Costa, M. G. de Oliveira, Colloids Surf. B 2015, 130, 182-191.

[3] M. H. Wu, S. Y. Yuan, H. J. Granger, J. Physiol. 2005, 563, $95-$ 104.

[4] F. C. Fang, J. Clin. Invest. 1997, 99, 2818-2825.

[5] L. Morbidelli, B. Bonavida, Therapeutic Application of Nitric Oxide in Cancer and Inflammatory Disorders, Academic Press, London, 2019.

[6] S. T. Gregg, Q. Yuan, R. E. Morris, B. Xiao, Mater. Today Commun. 2017, 12, 95-105.

[7] P. S. Wheatley, A. R. Butler, M. S. Crane, S. Fox, B. Xiao, A. G. Rossi, I. L. Megson, R. E. Morris, J. Am. Chem. Soc. 2006, 128, $502-509$.

[8] S. Fox, T. S. Wilkinson, P. S. Wheatley, B. Xiao, R. E. Morris, A. Sutherland, A. J. Simpson, P. G. Barlow, A. R. Butler, I. L. Megson, et al., Acta Biomater. 2010, 6, 1515-1521.

[9] M. L. Pinto, J. Rocha, J. R. B. Gomes, J. Pires, J. Am. Chem. Soc. 2011, 133, 6396-6402.

[10] M. L. Pinto, A. C. Fernandes, F. Antunes, J. Pires, J. Rocha, Microporous Mesoporous Mater. 2016, 229, 83-89.

[11] B. Xiao, P. S. Wheatley, X. Zhao, A. J. Fletcher, S. Fox, A. G. Rossi, I. L. Megson, S. Bordiga, L. Regli, K. M. Thomas, et al., J. Am. Chem. Soc. 2007, 129, 1203-1209.
[12] A. C. McKinlay, B. Xiao, D. S. Wragg, P. S. Wheatley, I. L. Megson, R. E. Morris, J. Am. Chem. Soc. 2008, 130, 1044010444.

[13] R. V. Pinto, F. Antunes, J. Pires, V. Graça, P. Brandão, M. L. Pinto, Acta Biomater. 2017, 51, 66-74.

[14] N. J. Hinks, A. C. McKinlay, B. Xiao, P. S. Wheatley, R. E. Morris, Microporous Mesoporous Mater. 2010, 129, 330-334.

[15] S. Diring, D. O. Wang, C. Kim, M. Kondo, Y. Chen, S. Kitagawa, K. I. Kamei, S. Furukawa, Nat. Commun. 2013, https://doi.org/ $10.1038 /$ ncomms 3684

[16] C. Kim, S. Diring, S. Furukawa, S. Kitagawa, Dalton Trans. 2015, 44, 15324-15333.

[17] M. Mowbray, X. Tan, P. S. Wheatley, A. G. Rossi, R. E. Morris, R. B. Weller, J. Invest. Dermatol. 2008, 128, 352-360.

[18] M. Neidrauer, U. K. Ercan, A. Bhattacharyya, J. Samuels, J. Sedlak, R. Trikha, K. A. Barbee, M. S. Weingarten, S. G. Joshi, J. Med. Microbiol. 2014, 63, 203-209.

[19] R. E. Morris, P. S. Wheatley, Angew. Chem. Int. Ed. 2008, 47, 4966-4981; Angew. Chem. 2008, 120, 5044-5059.

[20] M. L. Pinto, R. V. Pinto in Ther. Appl. Nitric Oxide Cancer Inflamm. Disord. (Eds.: L. Morbidelli, B. Bonavida), Academic Press, London, 2019, pp. 277-305.

[21] P. Horcajada, R. Gref, T. Baati, P. K. Allan, G. Maurin, P. Couvreur, G. Férey, R. E. Morris, C. Serre, Chem. Rev. 2012, 112, $1232-1268$.

[22] P. S. Wheatley, A. C. McKinlay, R. E. Morris in Zeolites and Related Materials: Trends Targets and Challenges Proceedings 4th Int. FEZA Conf. (Eds.: P. M. Antoine Gédéon, F. Babonneau), Elsevier, Amsterdam, 2008, pp. 441-446.

[23] J. Kim, B. C. Yung, W. J. Kim, X. Chen, J. Controlled Release 2017, 263, 223-230.

[24] B. Li, H.-M. Wen, W. Zhou, B. Chen, J. Phys. Chem. Lett. 2014, 5 , $3468-3479$.

[25] A. C. McKinlay, R. E. Morris, P. Horcajada, G. Férey, R. Gref, P. Couvreur, C. Serre, Angew. Chem. Int. Ed. 2010, 49, 6260-6266; Angew. Chem. 2010, 122, 6400-6406.

[26] T. Simon-Yarza, A. Mielcarek, P. Couvreur, C. Serre, Adv. Mater. 2018, 30, 1-15. Darticle number?

[27] W. Cai, J. Wang, C. Chu, W. Chen, C. Wu, G. Liu, Adv. Sci. 2019 , 6, 1801526.

[28] M. A. Luzuriaga, C. E. Benjamin, M. W. Gaertner, H. Lee, F. C. Herbert, S. Mallick, J. J. Gassensmith, Supramol. Chem. 2019, 31, $485-490$.

[29] S. Wang, T. Kitao, N. Guillou, M. Wahiduzzaman, C. MartineauCorcos, F. Nouar, A. Tissot, L. Binet, N. Ramsahye, S. Devautour-Vinot, et al., Nat. Commun. 2018, 9, 1660.

[30] F. Bonino, S. Chavan, J. G. Vitillo, E. Groppo, G. Agostini, C. Lamberti, P. D. C. Dietzel, C. Prestipino, S. Bordiga, Chem. Mater. 2008, 20, 4957-4968.

[31] S. R. Miller, E. Alvarez, L. Fradcourt, T. Devic, S. Wuttke, P. S. Wheatley, N. Steunou, C. Bonhomme, C. Gervais, D. Laurencin, et al., Chem. Commun. 2013, 49, 7773-7775.

[32] J. F. Eubank, P. S. Wheatley, G. Lebars, A. C. McKinlay, H. Leclerc, P. Horcajada, M. Daturi, A. Vimont, R. E. Morris, C. Serre, APL Mater. 2014, 2, 124112.

[33] A. C. Fernandes, F. Antunes, J. Pires, New J. Chem. 2013, 37, $4052-4060$.

[34] A. C. Fernandes, M. L. Pinto, F. Antunes, J. Pires, J. Mater. Chem. B 2015, 3, 3556-3563.

[35] A. C. Fernandes, M. L. Pinto, F. Antunes, J. Pires, RSC $A d v$. 2016, 6, 41195-41203.

[36] A. Fernandes, F. Antunes, M. L. Pinto, J. Pires, J. Mater. Chem. B 2013, 1, 3287-3294.

[37] A. C. McKinlay, J. F. Eubank, S. Wuttke, B. Xiao, P. S. Wheatley, P. Bazin, J.-C. Lavalley, M. Daturi, A. Vimont, G. De Weireld, et al., Chem. Mater. 2013, 25, 1592-1599.

[38] K. I. Hadjiivanov, Catal. Rev. Sci. Eng. 2000, 42, 71-144. 
[39] L. Qiu, D. Pang, C. Zhang, J. Meng, R. Zhu, F. Ouyang, Appl. Surf. Sci. 2015, 357, 189-196.

[40] M. Feelisch, D. Kubitzek, J. Werringloer in Methods in Nitric Oxide Research (Eds.: M. Feelisch, J. S. Stamler), Wiley, New York, 1996, pp. $455-478$.

[41] J. Dupré, P. Bazin, O. Marie, M. Daturi, X. Jeandel, F. Meunier, Appl. Catal. B 2016, 181, 534-541.

[42] G. Centi, S. Perathoner, Appl. Catal. A 1995, 132, 179-259.

[43] M. L. Pinto, A. C. Fernandes, J. Rocha, A. Ferreira, F. Antunes, J. Pires, J. Mater. Chem. B 2014, 2, 224-230.

[44] P. Horcajada, T. Chalati, C. Serre, B. Gillet, C. Sebrie, T. Baati, J. F. Eubank, D. Heurtaux, P. Clayette, C. Kreuz, et al., Nat. Mater. 2010, 9, $172-178$.

[45] S. Keskin, S. Kızılel, Ind. Eng. Chem. Res. 2011, 50, 1799-1812.

[46] D. Cattaneo, S. J. Warrender, M. J. Duncan, C. J. Kelsall, M. K. Doherty, P. D. Whitfield, I. L. Megson, R. E. Morris, RSC Adv. 2016, 6, 14059-14067.

[47] G. C. Brown, Biochim. Biophys. Acta Bioenerg. 1999, 1411, 351369.

[48] M. B. Witte, A. Barbul, Am. J. Surg. 2002, 183, 406-412.
[49] G. C. Brown, V. Borutaite, Free Radical Biol. Med. 2002, 33, $1440-1450$

[50] R. Zhan, S. Yang, W. He, F. Wang, J. Tan, J. Zhou, S. Yang, Z. Yao, J. Wu, G. Luo, PLoS One 2015, 10, e0121551.

[51] R. Zhan, W. He, F. Wang, Z. Yao, J. Tan, R. Xu, J. Zhou, Y. Wang, H. Li, J. Wu, et al., Sci. Rep. 2016, 6, 30687.

[52] V. Krischel, D. Bruch-Gerharz, C. Suschek, K.-D. Kröncke, T. Ruzicka, V. Kolb-Bachofen, J. Invest. Dermatol. 1998, 111, $286-$ 291.

[53] S. Kumar, M. K. Barthwal, M. Dikshit, Free Radical Biol. Med. 2010, 48, 851-861.

[54] A. Seabra, Nitric Oxide Donors: Novel Biomedical Applications and Perspectives, Elsevier Science, Amsterdam, 2017.

[55] M. R. Miller, I. L. Megson, Br. J. Pharmacol. 2007, 151, 305-321.

Manuscript received: October 14, 2019

Accepted manuscript online: January 17, 2020

Version of record online: $\mathbf{D} \mathbf{m}$, $\mathbf{W I}$ 


\section{Research Articles}

\section{NO Release}

R. V. Pinto, S. Wang, S. R. Tavares, J. Pires, F. Antunes, A. Vimont, G. Clet, M. Daturi, G. Maurin, C. Serre,*

M. L. Pinto*

III-III

Tuning Cellular Biological Functions Through the Controlled Release of NO from a Porous Ti-MOF

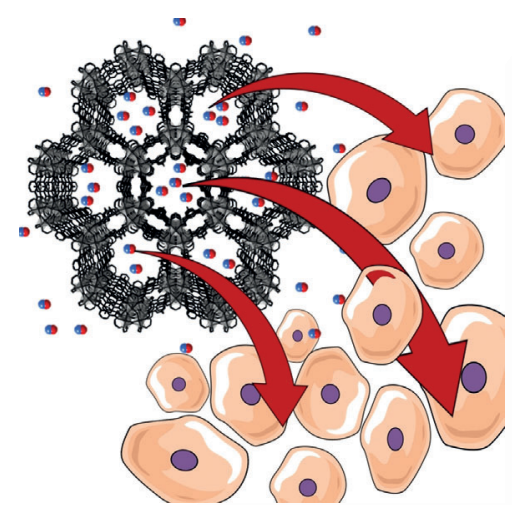

NO limit: The delivery of nitric oxide with a new titanium metal-organic framework allows the control of biological functions of cells, due to its adsorption/release mechanism based on the formation of nitrites bound to the titanium sites. The high storage capacity, biocompatibility and slow release and stability in biological media, render this material an exceptional vehicle for nitric oxide delivery.

Die Freisetzung von NO durch ein Timetallorganisches Gerüst ermöglicht aufgrund des NO-Speichermechanismus die Kontrolle der biologischen Funktionen von Zellen. Der Mechanismus beruht auf der Bildung von Nitriten, die an die Titanzentren gebunden sind. Die hohe Speicherkapazität, Biokompatibilität und die langsame Freisetzung und Stabilität in biologischen Medien machen dieses Material zu einem interessanten Werkzeug zur Abgabe von NO.
Tuning Cellular Biological Functions Through the Controlled Release of NO from a Porous Ti-MOF

\section{III-III}

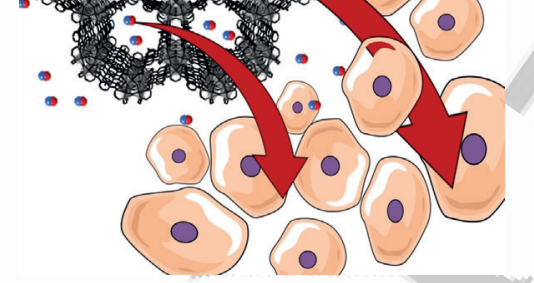

Share your work on social media! Angewandte Chemie has added Twitter as a means to promote your article. Twitter is an online microblogging service that enables its users to send and read short messages and media, known as tweets. Please check the pre-written tweet in the galley proofs for accuracy. If you, your team, or institution have a Twitter account, please include its handle @username. Please use hashtags only for the most important keywords, such as \#catalysis, \#nanoparticles, or \#proteindesign. The ToC picture and a link to your article will be added automatically, so the tweet text must not exceed 250 characters. This tweet will be posted on the journal's Twitter account (follow us @angew_chem) upon publication of your article in its final (possibly unpaginated) form. We recommend you to re-tweet it to alert more researchers about your publication, or to point it out to your institution's social media team. 
Please check that the ORCID identifiers listed below are correct. We encourage all authors to provide an ORCID identifier for each coauthor. ORCID is a registry that provides researchers with a unique digital identifier. Some funding agencies recommend or even require the inclusion of ORCID IDs in all published articles, and authors should consult their funding agency guidelines for details. Registration is easy and free; for further information, see http://orcid.org/.

Rosana V. Pinto

Dr. Sujing Wang

Dr. Sergio R. Tavares

Prof. João Pires

Prof. Fernando Antunes

Dr. Alexandre Vimont

Prof. Guillaume Clet

Prof. Marco Daturi

Prof. Guillaume Maurin

Dr. Christian Serre

Prof. Moisés L. Pinto http://orcid.org/0000-0003-3061-9632 